\title{
Some new additive and multiplicative Ramsey numbers
}

\author{
Neil Hindman* AND Dev Phulara
}

For $a, r \in \mathbb{N}$, the set of positive integers, define $F S P_{2}(a, r)$ (respectively $\left.S P_{2}(a, r)\right)$ to be the first $n \in \mathbb{N}$, if such exists, such that whenever $\{1,2, \ldots, n\}$ is $r$-colored, there exist $x$ and $y$ with $a \leq$ $x<y$ such that $\{x, y, x+y, x y\}$ is monochromatic (respectively $\{x+y, x y\}$ is monochromatic). If no such $n$ exists, the number is defined to be infinite. It is an old result of $\mathrm{R}$. Graham that $S P_{2}(a, 2)$ is finite for all $a$. With that exception, the only cases (with $r>1$ ) for which $F S P_{2}(a, r)$ or $S P_{2}(a, r)$ are known to be finite are those for which explicit values have been computed. In this paper, we provide exact values of $F S P_{2}(a, 2)$ for $a \leq 5$ (of which $F S P_{2}(1,2)$ and $F S P_{2}(2,2)$ were previously known). We provide exact values of $S P_{2}(a, 3)$ for $a \leq 9$ and exact values of $S P_{2}(a, 2)$ for $a \leq 105$. We also compute upper and lower bounds for $S P_{2}(a, 2)$.

2010 Mathematics Subject Classifications: 05D10.

\section{Introduction}

In [1, Theorem 2.6], it was shown that if $r \in \mathbb{N}$ and $\mathbb{N}$ is $r$-colored, there exist sequences $\left\langle x_{n}\right\rangle_{n=1}^{\infty}$ and $\left\langle y_{n}\right\rangle_{n=1}^{\infty}$ such that $F S\left(\left\langle x_{n}\right\rangle_{n=1}^{\infty}\right) \cup F P\left(\left\langle y_{n}\right\rangle_{n=1}^{\infty}\right)$ is monochromatic. Here $F S\left(\left\langle x_{n}\right\rangle_{n=1}^{\infty}\right)=\left\{\sum_{t \in F} x_{t}: F \in \mathcal{P}_{f}(\mathbb{N})\right\}$ and $F P\left(\left\langle y_{n}\right\rangle_{n=1}^{\infty}\right)=\left\{\prod_{t \in F} y_{t}: F \in \mathcal{P}_{f}(\mathbb{N})\right\}$, where $\mathcal{P}_{f}(\mathbb{N})$ is the set of finite nonempty subsets of $\mathbb{N}$. At that time, it was not known whether one could always choose one sequence $\left\langle x_{n}\right\rangle_{n=1}^{\infty}$ with $F S\left(\left\langle x_{n}\right\rangle_{n=1}^{\infty}\right) \cup F P\left(\left\langle x_{n}\right\rangle_{n=1}^{\infty}\right)$ monochromatic. However, it was shown in [3] that there is a 7-coloring of $\mathbb{N}$ such that there is no sequence $\left\langle x_{n}\right\rangle_{n=1}^{\infty}$ with $P S\left(\left\langle x_{n}\right\rangle_{n=1}^{\infty}\right) \cup P P\left(\left\langle x_{n}\right\rangle_{n=1}^{\infty}\right)$ monochromatic, where $P S\left(\left\langle x_{n}\right\rangle_{n=1}^{\infty}\right)=\left\{x_{n}+x_{m}: n \neq m\right\}$ and $P P\left(\left\langle x_{n}\right\rangle_{n=1}^{\infty}\right)=$ $\left\{x_{n} x_{m}: n \neq m\right\}$.

Given a finite sequence $\left\langle x_{t}\right\rangle_{t=1}^{k}$, the notations $F S\left(\left\langle x_{t}\right\rangle_{t=1}^{k}\right), F P\left(\left\langle x_{t}\right\rangle_{t=1}^{k}\right)$, $P S\left(\left\langle x_{t}\right\rangle_{t=1}^{k}\right)$, and $P P\left(\left\langle x_{t}\right\rangle_{t=1}^{k}\right)$ have their obvious meanings. The first author of this paper has maintained for a few decades that the following is a fact.

*This author acknowledges support received from the National Science Foundation via Grant DMS-1160566. 
Let $r, k \in \mathbb{N}$ and let $\mathbb{N}$ be $r$-colored. There exists a sequence $\left\langle x_{t}\right\rangle_{t=1}^{k}$ such that $F S\left(\left\langle x_{t}\right\rangle_{t=1}^{k}\right) \cup F P\left(\left\langle x_{t}\right\rangle_{t=1}^{k}\right)$ is monochromatic.

It should be understood that he has not claimed that he can prove this fact. The only case (with $r>1$ ) with a proof is $r=k=2$. And this is a computer generated result. Sometime in the mid 1970's, R. Graham used a computer program to verify that if $\{1,2, \ldots, 252\}$ is 2 -colored, then there exist $x \neq y$ such that $\{x, y, x+y, x y\}$ is monochromatic and that the corresponding statement fails for $\{1,2, \ldots, 251\}$. He noted that the fact that $x=1$ is allowed is crucial because, if $y$ and 1 are the same color, then $y+1$ must be the opposite color. Accordingly, one is led to define $F S P_{2}(a, r)$ as in the abstract and ask whether one can establish that $F S P_{2}(a, r)$ is finite for all $a$ and $r$.

In [1], the fact that $F S P_{2}(1,2)=252$ was verified and it was established that $F S P_{2}(2,2)=990$. These results were obtained using a Fortran program on the IBM mainframe computer in use at SUNY Binghamton in 1976. The result for $F S P_{2}(1,2)$ took approximately two seconds of computer time and the result for $F S P_{2}(2,2)$ took approximately four minutes of computer time. The author of [1] did not try to find $F S P_{2}(3,2)$, feeling that the amount of computer time required would not be feasible. In 2012, running a Pascal program on a desktop computer with an Intel Cor2Duo CPU E8400 processor operating at $3.00 \mathrm{GHz}$, the result for $F S P_{2}(2,2)$ took less than 2 seconds of computer time. And the fact that $F S P_{2}(3,2)=3150$ was established using less than 52 seconds of computer time. We no longer have the program used in Binghamton, and we were able to significantly improve the efficiency of the current program by some modifications, but we suspect that the main difference in time consumption comes from improvement in processor speeds.

In Section 2 of this paper, we discuss the basic coloring algorithm used to compute $F S P_{2}(a, 2), S P_{2}(a, 2)$, and $S P_{2}(a, 3)$, as well as the limits on extending the results to higher values of $a$.

In the case of $S P_{2}(a, 2)$, we have a good deal of additional information. As we mentioned in the abstract, $\mathrm{R}$. Graham proved that $S P_{2}(a, 2)$ is finite for all $a$. (This proof was presented with his permission as [2, Theorem 3.3].) We have computed the upper bounds that are provided by this original proof and also have computed a very slight improvement. Both of these bounds are on the order of $\frac{8}{9} a^{4}$. We also prove a lower bound on the order of $a^{3}$. These results are presented in Section 3.

We conclude this introduction by presenting the exact numbers that we have obtained. As we have remarked, the fact that $F S P_{2}(1,2)=252$ is due 
Table 1: Values of $F S P_{2}(a, 2)$

\begin{tabular}{rr}
\hline$a$ & $F S P_{2}(a, 2)$ \\
\hline 1 & \\
2 & 252 \\
3 & 990 \\
4 & 3150 \\
5 & 5600 \\
\hline
\end{tabular}

Table 2: Values of $S P_{2}(a, 3)$

\begin{tabular}{rr}
\hline$a$ & $S P_{2}(a, 3)$ \\
\hline 1 & 100 \\
2 & 216 \\
3 & 774 \\
4 & 3504 \\
5 & 11100 \\
6 & 28260 \\
7 & 62034 \\
8 & 122304 \\
9 & 222264 \\
\hline
\end{tabular}

to R. Graham. The fact that $S P_{2}(1,3)=100$ was established by D. Tang in [4], where a detailed proof that any 3 -coloring of $\{1,2, \ldots, 100\}$ has a monochromatic $\{x+y, x y\}$ with $x<y$ was presented.

\section{The coloring algorithm}

Flow charts for the basic coloring algorithm used to compute values for $F S P_{2}(a, 2), S P_{2}(a, 2)$, and $S P_{2}(a, 3)$ are in Figures 1, 2, 3, and either 4 (for $F S P_{2}(a, 2)$ and $S P_{2}(a, 2)$ ) or 5 (for $S P_{2}(a, 3)$ ). For $S P_{2}(a, r)$, one is dealing with a graph with vertex set $V=\{a, a+1, \ldots, \max \}$ and edges of the form $\{x+y, x y\}$ where $a \leq x<y$. If one can $r$-color $V$ with no monochromatic edges, then $S P_{2}(a, r)>$ max. In discussing $F S P_{2}(a, 2)$ we will use hypergraph terminology, so that an "edge" is a set of the form $\{x, y, x+y, x y\}$ where $a \leq x<y$.

For each of the calculations, one inputs the numbers $a$ and $m$. If there is an edge-free coloring of $V$, the algorithm will find one. If there is none, the algorithm amounts to a proof by cases that no such coloring exists.

The "preliminary calculations" referred to in Figure 1 involve such things as computing the degrees of vertices and sorting so that the higher degree 
Table 3: Values of $S P_{2}(a, 2)$

\begin{tabular}{rrrrrr}
\hline$a$ & $S P_{2}(a, 2)$ & $a$ & $S P_{2}(a, 2)$ & $a$ & $S P_{2}(a, 2)$ \\
\hline 1 & & & & & \\
2 & 8 & 36 & 68688 & 71 & 514182 \\
3 & 24 & 37 & 80771 & 72 & 523584 \\
4 & 54 & 38 & 83752 & 73 & 554216 \\
5 & 128 & 39 & 86697 & 74 & 564028 \\
6 & 250 & 40 & 96000 & 75 & 573750 \\
7 & 432 & 41 & 109265 & 76 & 583376 \\
8 & 686 & 42 & 112896 & 77 & 592900 \\
9 & 1024 & 43 & 116487 & 78 & 638820 \\
10 & 1377 & 44 & 120032 & 79 & 661546 \\
11 & 2662 & 46 & 152352 & 81 & 721710 \\
12 & 3168 & 47 & 156839 & 82 & 766536 \\
13 & 3887 & 48 & 161280 & 83 & 778457 \\
14 & 4312 & 49 & 180075 & 84 & 790272 \\
15 & 5625 & 50 & 185000 & 85 & 867000 \\
16 & 6912 & 51 & 189873 & 86 & 880124 \\
17 & 8959 & 52 & 205504 & 87 & 893142 \\
18 & 9720 & 53 & 210675 & 88 & 906048 \\
19 & 11552 & 54 & 215784 & 89 & 926757 \\
20 & 12400 & 55 & 235950 & 90 & 939600 \\
21 & 14553 & 56 & 241472 & 91 & 1010282 \\
22 & 16456 & 57 & 272916 & 92 & 1100320 \\
23 & 20102 & 58 & 292668 & 93 & 1115721 \\
24 & 21312 & 59 & 299366 & 94 & 1139844 \\
25 & 26875 & 60 & 306000 & 95 & 1155200 \\
26 & 28392 & 61 & 320006 & 96 & 1170432 \\
27 & 29889 & 62 & 326740 & 97 & 1213761 \\
28 & 32928 & 63 & 333396 & 98 & 1229312 \\
29 & 38686 & 64 & 360448 & 99 & 1244727 \\
30 & 40500 & 65 & 367575 & 100 & 1280000 \\
31 & 48050 & 66 & 400752 & 101 & 1366934 \\
32 & 50176 & 67 & 430944 & 102 & 1383732 \\
33 & 52272 & 68 & 439280 & 103 & 1495869 \\
34 & 60112 & 69 & 447534 & 104 & 1514240 \\
35 & 66150 & 70 & 455700 & 105 & 1543500 \\
\hline & & & & &
\end{tabular}

vertices come first. Here also some tables may be computed that allow one to quickly find which vertices a given vertex is connected to. In the case of the computation of $S P_{2}(a, 3)$, one enters by hand the vertices of a triangle, assigning each such vertex to one color. For example, when $a=3$, the values 


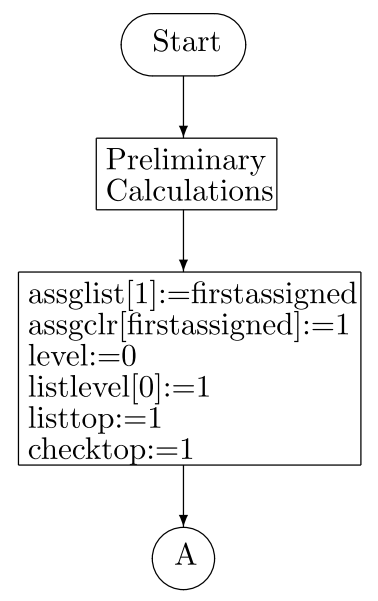

Figure 1: Start of Coloring Algorithm.

Table 4: Smallest Triangles

\begin{tabular}{cc}
\hline$a$ & Triangle \\
\hline & \\
4 & $\{59,220,864\}$ \\
5 & $\{94,445,2200\}$ \\
6 & $\{137,786,4680\}$ \\
7 & $\{188,1267,8820\}$ \\
8 & $\{247,1912,15232\}$ \\
9 & $\{314,2745,24624\}$ \\
\hline
\end{tabular}

32,87 , and 252 are vertices of a triangle. $(32=3+29$ and $87=3 \cdot 29$ so $\{32,87\}$ is an edge. $32=14+18$ and $252=14 \cdot 18$ so $\{32,252\}$ is an edge. $87=3+84$ and $252=3 \cdot 84$ so $\{87,252\}$ is an edge.) Of these, only $\{32,252\}$ remains an edge when $a=4$. The smallest triangles for $a=3$ through 9 are given in Table 4.

There are three variables and three arrays that are updated throughout the program. The array entry assgclr[i] is 0 if $i$ has not been assigned to a color, and otherwise is the number of the color to which $i$ has been assigned; assglist [i] is the $i^{\text {th }}$ vertex assigned; and listlevel [i] is the address in assglist of the first item assigned when level=i. The variable level records the number of free assignments made; listtop is the address in assglist of the last assignment made; and checktop is the address in assglist of the last entry which has been checked for forcing or contradictions resulting from its assignment. 


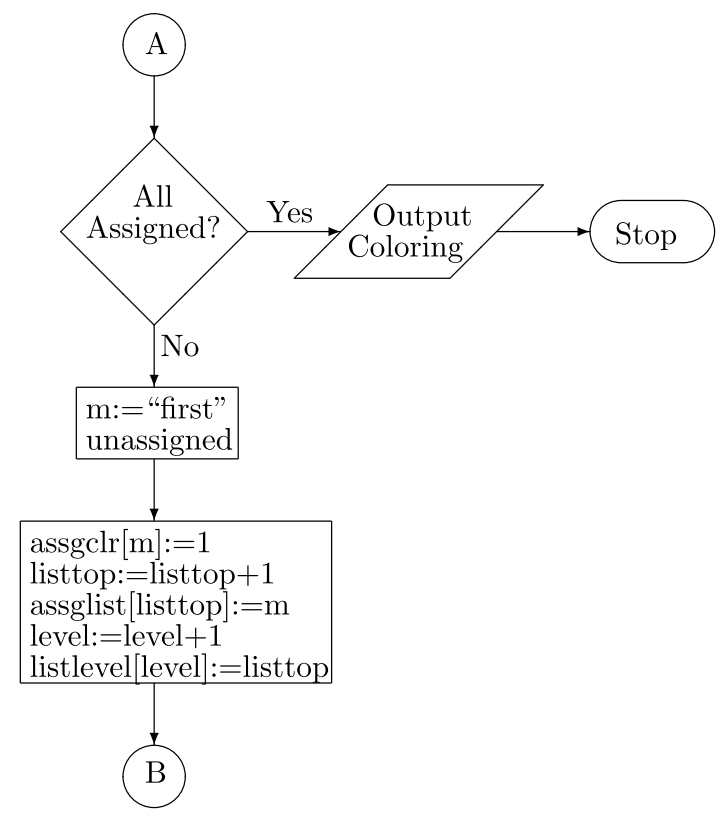

Figure 2: Free Assignments.

In Figure 2, the "first" unassigned is one of the highest degree vertices which have not been assigned. One such is assigned to color 1 . Then, as described in Figure 3, one checks whether this assignment forces a monochromatic edge. If not, one finds all vertices which are forced to some other color (called "kolor" in Figure 3). The process is iterated to see what is forced by each of the new assignments.

If there is a monochromatic edge, then one proceeds to the "contradiction" segment. What is done here depends on whether one is considering 2-colorings (Figure 4) or 3-colorings (Figure 5). In either case, let $\mathrm{k}$ be the vertex which was freely assigned at the current value of level and let color be the color to which it was assigned. In the case of 2-colorings, necessarily color $=1$, and since this assignment led to a contradiction, $\mathrm{k}$ is forced to color 2 at the previous value of level (unless level $=0$, in which case no good coloring is possible).

In the case of 3 -colorings, if color $=1$, then one needs to consider the assigment of $\mathrm{k}$ to color 2 . If color $=2$, then since both the assignment of $\mathrm{k}$ to color 1 and to color 2 has led to a contradiction, $\mathrm{k}$ is forced to color 3 at the previous value of level (again unless level $=0$, in which case no good coloring is possible). 


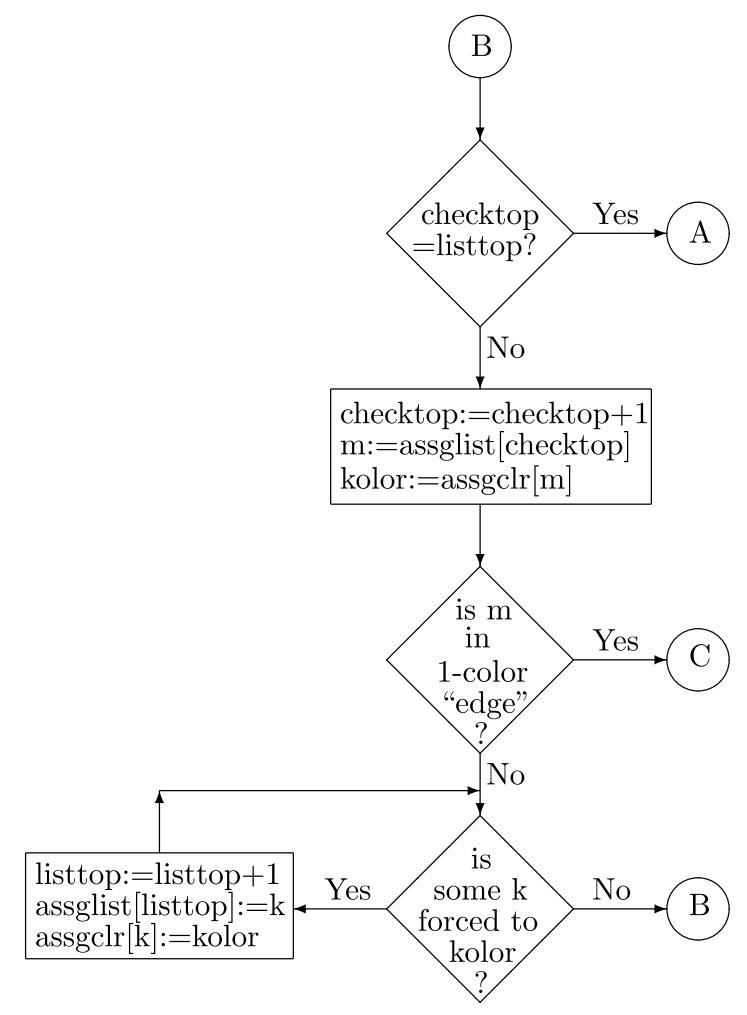

Figure 3: Forcing.

In terms of time consumption, the most critical part of the algorithm is in Figure 3 where one determines whether $\mathrm{m}$ is in a monochromatic edge and whether the assignment of $\mathrm{m}$ to kolor forces some other vertex to a different color. Consider for example the program for computing $S P_{2}(a, 3)$. Computing from scratch each time whether there is an edge from $\mathrm{m}$ to $\mathrm{k}$ would, of course, be very time consuming. Initially, among the preliminary calculations, we computed a $\{a, a+1, a+2, \ldots, \max \} \times\{a, a+1, a+2, \ldots, \max \}$ array edges with edges $[i, j]=1$ if there is an edge between $i$ and $j$ and edges $[i, j]=0$ otherwise. One then checked individually for each value of $\mathrm{k}$ whether there is an edge from $\mathrm{m}$ to $\mathrm{k}$. Running this version of the algorithm on a laptop computer with an Intel Cor2Duo CPU P8400 processor operating at $2.26 \mathrm{GHz}$ with $a=6$ and $\max =28259$, it took 246 seconds to find a coloring with no edges, and with $\max =28260$, it took 350 seconds to determine that all colorings have a monochromatic edge.

Motivated partly by the fact that the array edges was taking up a huge amount of RAM as $a$ increased, we changed edges to a one dimensional array 


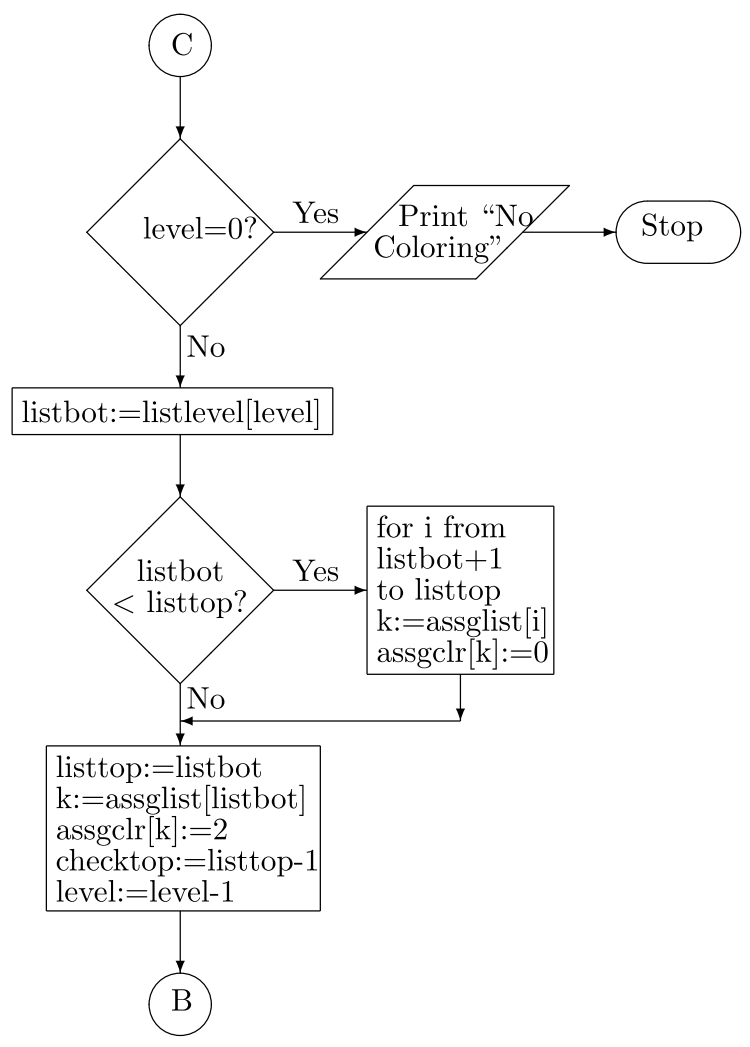

Figure 4: Contradiction for 2-coloring.

(with size twice the total number of edges) and introduced a new $\{a, a+1, a+$ $2, \ldots, \max \} \times\{1,2\}$ array inedges. We set inedges $[i, 1]=0$ if the degree of $i$ is 0 . Otherwise, we set inedges $[i, 1]=k$ and inedges $[i, 2]=m$ where edges $[k]$, edges $[k+1], \ldots$, edges $[\mathrm{m}]$ are the other ends of edges with $i$. This significantly reduced the amount of RAM being used and allowed to only check values of $\mathrm{k}$ which do form an edge with $\mathrm{m}$. Running the revised program on the same laptop with $a=6$ and $\max =28259$, it took 7 seconds to find a coloring with no edges, and with $\max =28260$, it took 5 seconds to determine that all colorings have a monochromatic edge.

A word about our confidence in our results is in order. In principle, when the program declares that there are no good colorings, one could have the computer print the cases considered and use those to write out a proof. This was in fact done in [1, Theorem 4.3]. But for larger numbers, this is not feasible. What we are very confident of is the fact that each of the listed 


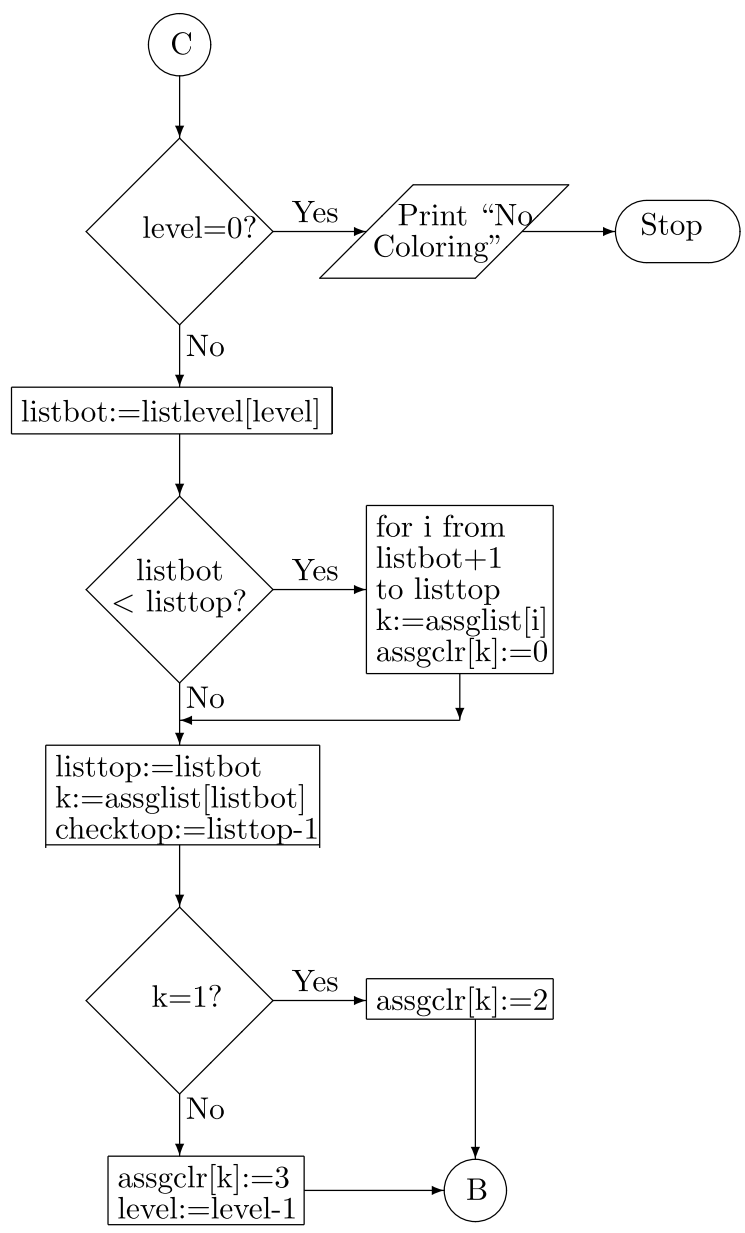

Figure 5: Contradiction for 3-coloring.

numbers is at least as large as stated. This is because we had the program in each instance produce a file which had the colors to which each number was assigned. This file was then used as input for a separate program which, with a very simple and transparent algorithm, verified that the coloring had no monochromatic edges.

We conclude this section with a brief discussion of what made us stop when we did. For the computation of $F S P_{2}(a, 2)$, the reason was simply time consumption. Running on the same laptop mentioned above, it took 3 hours, 47 minutes, and 19 seconds to find a 2-coloring of $\{5,6, \ldots, 14363\}$ with no $x<y$ such that $\{x, y, x+y, x y\}$ is monochromatic, and it took 3 hours, 54 
minutes, and 7 seconds to show that any 2 -coloring of $\{5,6, \ldots, 14364\}$ does have a monochromatic edge. This contrasts with times of 40 seconds for $\{4,5, \ldots, 5599\}$ and 13 minutes and 44 seconds for $\{4,5, \ldots, 5600\}$. It seems clear that finding $\mathrm{FSP}_{2}(6,2)$ using this program is not feasible. (Don't forget that the program needs to be run once for each value of max that is tested.)

For the computation of $S_{2}(a, 3)$, the constraint was available RAM. The revised program mentioned three paragraphs above required twice the storage (as 4 byte numbers) as the number of edges. The number of edges in $\{7,8, \ldots, 62033\}$ is 194985 . The number of edges in $\{8,9, \ldots, 122303\}$ is 408521. The number of edges in $\{9,10, \ldots, 222263\}$ is 781075 .

For the computation of $S P_{2}(a, 2)$, the constraint was largely available RAM, mixed in with a feeling of diminishing returns. The main reason we continued all the way to 105 was the fact, which we will discuss further in the next section, that in each case we kept finding that $S P_{2}(a, 2)$ is divisible by $a^{2}$.

\section{Bounds for $S P_{2}(a, 2)$}

In this section, we compute the upper bound on $S P_{2}(a, 2)$ which results from Graham's original proof that the number is finite, and establish a slight improvement on that bound. Both of these upper bounds are on the order of $\frac{8}{9} a^{4}$. And we establish a lower bound of $a^{2}(a+\lfloor 2 \sqrt{a}\rfloor)$.

For $a \in\{3,4,5,6,7,8,11\}, S P_{2}(a, 2)=2 a^{3}$ and for all computed cases with $a>2, S P_{2}(a, 2) \leq 2 a^{3}$. We have not been able to prove that $2 a^{3}$ is an upper bound.

We begin with the very simple proof of our lower bound.

Theorem 3.1. Let $a \in \mathbb{N}$. Then $S P_{2}(a, 2) \geq a^{2}(a+\lfloor 2 \sqrt{a}\rfloor)$.

Proof. The conclusion holds for $a=1$ and $a=2$, so assume that $a \geq 3$. Let $k=a+\lfloor 2 \sqrt{a}\rfloor$ and note that $\left(\frac{a+k}{2}\right)^{2} \leq a(k+1)$. Let

$$
A_{1}=\{a, a+1, \ldots, a+k\} \cup\left\{a(k+1), a(k+1)+1, \ldots, a^{2} k-1\right\}
$$

and let $A_{2}=\{a+k+1, a+k+2, \ldots, a(k+1)-1\}$. We need to show that there do not exist $x$ and $y$ with $a \leq x<y$ and $i \in\{1,2\}$ such that $\{x+y, x y\} \subseteq A_{i}$. So suppose instead we have such $x, y$, and $i$.

If $x+y \geq a(k+1)$, then, since $x \geq a, x y \geq a(x+y-a) \geq a^{2} k$ so $x y \notin\left(A_{1} \cup A_{2}\right)$. Suppose $x+y \in\{a, a+1, \ldots, a+k\}$. Then $x y \geq a(a+1)$ and $a(a+1)>a+k$ since $a>2$. Also $x y<\left(\frac{a+k}{2}\right)^{2} \leq a(k+1)$ so $x y \notin A_{1}$.

Finally suppose that $x+y \in A_{2}$. Then $x y \geq a(x+y-a) \geq a(k+1)$ so $x y \notin A_{2}$. 
Now we present the upper bound which is given by Graham's original argument.

Theorem 3.2. Let $a \in \mathbb{N}$ and let $t=\left\lfloor\frac{a}{3}\right\rfloor$.

(1) If $a=3 t$, then $S P_{2}(a, 2) \leq 72 t^{4}+72 t^{3}+6 t^{2}-6 t-4$.

(2) If $a=3 t+1$, then $S P_{2}(a, 2) \leq 72 t^{4}+216 t^{3}+222 t^{2}+90 t+8$.

(3) If $a=3 t+2$, then $S P_{2}(a, 2) \leq 72 t^{4}+288 t^{3}+420 t^{2}+264 t+56$.

Proof. We shall establish (1), the other proofs being very similar. The case $t=1$ holds by hand computation, so assume $t>1$. Let $k=t^{2}+3 t$. We are claiming that $S P_{2}(a, 2) \leq 2(k-2)(k+1)$. Let $\varphi:\{1,2, \ldots, 2(k-2)(k+1)\} \rightarrow$ $\{1,2\}$.

Case 1. $\varphi(3(3 t+1))=(3(3 t+2))=\ldots=\varphi(3 k)$. Let $x=3 t$ and let $y=6 t+3$. Then $a=x<y, x+y=3(3 t+1)$, and $x y=3 k<2(k-2)(k+1)$.

Case 2. Not case 1. Pick $d \in\{3 t, 3 t+1, \ldots, k-2\}$ such that $\varphi(3 d+3) \neq$ $\varphi(3 d+6)$. Then $d(2 d+6)=2 d(d+3)$. If $\varphi(d(2 d+6))=\varphi(3 d+6)$, let $x=d$ and let $y=2 d+6$. If $\varphi(2 d(d+3))=\varphi(3 d+3)$, let $x=d+3$ and $y=2 d$. Since $t>1, x<y$.

Graham's original argument was based on the fact that if all of the multiples of 3 are the same color, then one trivially gets arbitrarily large $x<y$ such that $x+y$ and $x y$ are the same color. Our slight improvement is based on the fact that if all of the elements which are not multiples of 3 are the same color, the same conclusion holds.

Theorem 3.3. Let $a \in \mathbb{N}$ and let $t=\left\lfloor\frac{a}{3}\right\rfloor$.

(1) If $a=3 t$, then $S P_{2}(a, 2) \leq 72 t^{4}+72 t^{3}-6 t^{2}-12 t$.

(2) If $a=3 t+1$, then $S P_{2}(a, 2) \leq 72 t^{4}+144 t^{3}+96 t^{2}+24 t$.

(3) If $a=3 t+2$, then $S P_{2}(a, 2) \leq 72 t^{4}+216 t^{3}+234 t^{2}+108 t+8$.

Proof. We shall establish (2), the other proofs being very similar. (Again, for (1), the case $t=1$ needs separate verification.) Let $k=6 t^{2}+6 t$. We are claiming that $S P_{2}(a, 2) \leq 2 k(k+2)$. Let $\varphi:\{1,2, \ldots, 2 k(k+2)\} \rightarrow\{1,2\}$.

Case 1. There is some $m \in\{a, a+1, \ldots, k\}$ such that $\varphi(3 m+1) \neq$ $\varphi(3 m+2)$. Then $2 m(m+1)<2 k(k+2)$. If $\varphi(2 m(m+1))=\varphi(3 m+1)$, let $x=m+1$ and $y=2 m$. If $\varphi(m(2 m+2))=\varphi(3 m+2)$, let $x=m$ and $y=2 m+2$.

Case 2. There is some $m \in\{a, a+1, \ldots, k\}$ such that $\varphi(3 m+2) \neq$ $\varphi(3 m+4)$. Then $2 m(m+2) \leq 2 k(k+2)$. If $\varphi(2 m(m+2))=\varphi(3 m+2)$, let $x=m+2$ and $y=2 m$. If $\varphi(m(2 m+4))=\varphi(3 m+4)$, let $x=m$ and $y=2 m+2$. 
Case 3. For all $m \in\{a, a+1, \ldots, k\}, \varphi(3 m+1)=\varphi(3 m+2)$ and for all $m \in\{a, a+1, \ldots, k\}, \varphi(3 m+2)=\varphi(3 m+4)$. Then $\varphi(3 a+1)=\varphi(3 k+4)$. Let $x=a+1$ and $y=2 a$.

As we noted earlier, in all computed cases, $S P_{2}(a, 2)$ is divisible by $a^{2}$. As $a$ increases, this seems less likely to be a random occurrence. But we have been unable even to prove that $S P_{2}(a, 2)$ is divisible by $a$.

Conjecture 3.4. For all $a \in \mathbb{N}, S P_{2}(a, 2)$ is divisible by $a$.

We close with two experimental observations. The first, which allowed us to compute $S_{2}(a, 2)$ with very small time consumption, is the fact that in each case the graph with edges $\{x+y, x y\}$ for $a \leq x<y$ consisted of the component of $2 a+1$ together with a graph which is trivially bipartite. (As a consequence, to verify on the laptop described previously that $\{105,106, \ldots, 1543500\}$ could not be two-colored without monochromatic edges took less than 13 seconds of processor time while to show that the graph on $\{105,106, \ldots, 1543499\}$ is bipartite took 6 seconds of processor time.) In most cases the remainder either consisted of a large number of degree 0 vertices and a few (at most 15) isolated edges, or it consisted of the degree 0 vertices, some isolated edges, and one or two disjoint stars. The most complicated configuration of the remainder after deleting the component of $2 a+1$ occurred when $a=53$. Then this remainder had 6 isolated edges and stars centered at 129, 130, 131, and 133 whose centers had degrees of $12,12,13$, and 14 respectively. The stars centered at 129 and 130 had the vertex 4104 in common; the stars centered at 130 and 131 had the vertex 4200 in common; the stars centered at 131 and 133 had the vertex 4104 in common; and the stars centered at 129 and 133 had the vertex 4158 in common.

Our second experimental observation is that in most, but not all, cases the 2-coloring $A_{1}, A_{2}$ of $\left\{a, a+1, \ldots, S P_{2}(a, 2)-1\right\}$ which our program found was very similar to the coloring in Theorem 3.1. That is, there was some number $m$ such that, letting $k=S P_{2}(a, 2)$, letting $b=a+\frac{k}{a}$, and letting $B=\{x y: a \leq x<y, x+y \leq m$, and $x y \geq b\}$, one had

$$
A_{1}=(\{a, a+1, \ldots, m\} \cup\{b, b+1, \ldots, k-1\}) \backslash B
$$

and $A_{2}=\{m+1, m+2, \ldots, b-1\} \cup B$.

\section{References}

[1] N. Hindman (1979). Partitions and sums and products of integers. Trans. Amer. Math. Soc. 247 227-245. MR0517693 
[2] N. Hindman (1979). Simultaneous idempotents in $\beta \mathbb{N} \backslash \mathbb{N}$ and finite sums and products in $\mathbb{N}$. Proc. Amer. Math. Soc. 77 150-154. MR0539649

[3] N. Hindman (1984). Partitions and pairwise sums and products. J. Comb. Theory (Series A) 37 46-60. MR0749511

[4] D. Tang (1997). Separating Sums From Products in $\mathbb{N}$. Ph.D. Dissertation, Howard University.

Neil Hindman

DePARTMENT of Mathematics

HOWARD UNIVERSITY

WASHINGTON, DC 20059

USA

E-mail address: nhindman@aol.com

URL: http://nhindman.us

Dev Phulara

Department of Mathematics

HOWARD UNIVERSITY

WASHINGTON, DC 20059

USA

E-mail address: phulara@comcast.net

Received October 4, 2012 